\title{
DECOUPLING PHASE VARIATIONS IN MULTI-PLANET SYSTEMS
}

\author{
Stephen R. Kane and Dawn M. Gelino \\ NASA Exoplanet Science Institute, Caltech, MS 100-22, 770 South Wilson Avenue, Pasadena, CA 91125, USA; skane@ipac.caltech.edu \\ Received 2012 August 28; accepted 2012 November 28; published 2012 December 21
}

\begin{abstract}
Due to the exquisite photometric precision, transiting exoplanet discoveries from the Kepler mission are enabling several new techniques of confirmation and characterization. One of these newly accessible techniques analyzes the phase variations of planets as they orbit their stars. The predicted phase variation for multi-planet systems can become rapidly complicated and depends upon the period, radius, and albedo distributions for planets in the system. Here we describe the confusion that may occur due to short-period terrestrial planets and/or non-transiting planets in a system, which can add high-frequency correlated noise or low-frequency trends to the data stream. We describe these sources of ambiguity with several examples, including that of our solar system. We further show how decoupling of these signals may be achieved with application to the Kepler-20 and Kepler-33 multi-planet systems.
\end{abstract}

Key words: planetary systems - techniques: photometric

\section{INTRODUCTION}

The past few decades represent an extraordinary period of growth in our knowledge of planetary systems. Whereas previously we used the single data point of our own solar system to derive theories of planetary formation, we now have a diverse range of planetary systems to draw upon. We are gaining an improved understanding of the distribution of planetary properties such as period, mass, eccentricity, radius, and multiplicity. These last two have been aided in no small part by the significant discoveries of the Kepler mission, which has revealed numerous cases of transiting planets in multi-planet systems (Borucki et al. 2011a, 2011b; Batalha et al. 2012). Many of these are tightly packed systems of multiple planets within a relatively small period range, examples of which include Kepler-11 (Lissauer et al. 2011a), Kepler-20 (Fressin et al. 2012; Gautier et al. 2012), and Kepler-33 (Lissauer et al. 2012). Lissauer et al. (2011b) and Lissauer et al. (2012) point out that these multi-planet systems comprise a large fraction of the total candidates detected and are far less likely to be due to false-positives.

The precision of the Kepler photometry has also allowed the investigation of out-of-transit variations that are phased with the planetary orbit. These include ellipsoidal variations of the host star induced by the planet (Jackson et al. 2012; Pfahl et al. 2008), Doppler boosting or beaming (Mazeh et al. 2012; Shporer et al. 2011), and reflected light from the planet (Kane \& Gelino 2010, 2011, 2012). A combination of these effects has been detected in several cases, such as HAT-P-7b which was observed in Kepler data to have signatures of reflected light as well as ellipsoidal variations (Welsh et al. 2010). These effects have almost exclusively been considered for single-planet systems, usually in very short period orbits where these effects will be higher in amplitude.

Here we consider the confusion that can result from multiplanet systems when attempting to measure the photometric phase variations resulting from reflected light. Since there will be a variety of periods, radii, and albedos, the contribution of each planet to the total phase variations may encompass a large range of amplitudes depending on how these values are distributed. The result of this is that certain planetary configurations will have "rogue" phase variation components that masquerade as correlated noise in the data. We describe a decoupling procedure for these systems to disentangle the signatures of the individual planets. We discuss the implications for the solar system as an externally observed exosystem and apply the method to the Kepler multi-planet systems of Kepler-20 and Kepler-33.

\section{PHOTOMETRIC PHASE VARIATIONS}

The phase variations of exoplanets are described in detail from numerous sources, such as those mentioned in Section 1. We refer the reader to Kane \& Gelino (2010) and Kane \& Gelino (2011) since we will be adopting that particular formalism.

The flux ratio of an exoplanet to the host star as observed from Earth is given by the following expression:

$$
\epsilon(\alpha, \lambda) \equiv \frac{f_{p}(\alpha, \lambda)}{f_{\star}(\lambda)}=A_{g}(\lambda) g(\alpha, \lambda) \frac{R_{p}^{2}}{r^{2}},
$$

where $\lambda$ is the wavelength of the observations, $A_{g}(\lambda)$ is the geometric albedo, $g(\alpha, \lambda)$ is the phase function, and $R_{p}$ is the radius of the planet. The phase angle of the planet, $\alpha$, is defined to be zero when the planet is at superior conjunction. Here we also allow for orbital eccentricity by using the time-dependent star-planet separation, $r$, given by

$$
r=\frac{a\left(1-e^{2}\right)}{1+e \cos f}
$$

where $a$ is the semi-major axis and $e$ is the orbital eccentricity. Notice that Equation (1) contains three major components: the geometric albedo, the phase function, and the inverse-square relation to the star-planet separation. For a given phase function, the flux ratio is proportional to the albedo, the square of the planetary radius, and the inverse square of the separation. This is equivalent to being approximately proportional to $P^{3 / 4}$, where $P$ is the orbital period. In other words,

$$
\epsilon(\alpha, \lambda) \propto A_{g} \times R_{p}^{2} \times P^{3 / 4} .
$$

The radius is clearly the dominant component which influences the amplitude of the phase variation. As we will see, this is an important factor when assessing the signatures of multi-planet systems, particularly the tightly packed systems detected by the Kepler mission. 


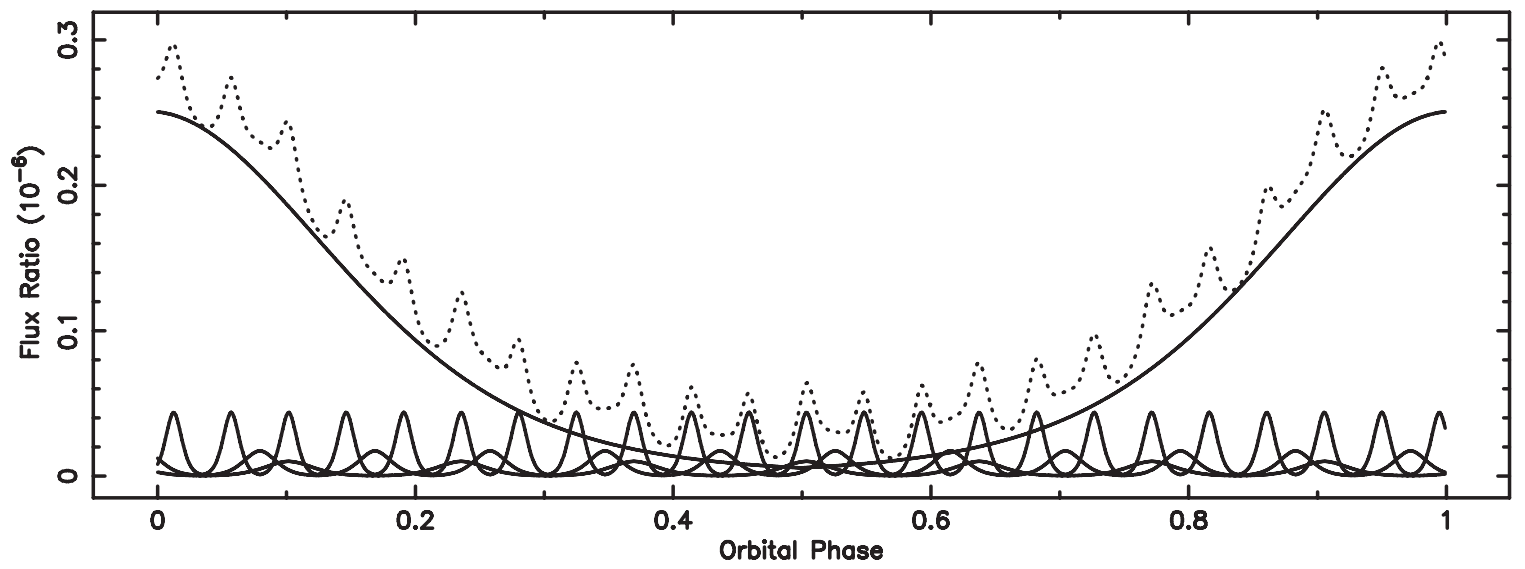

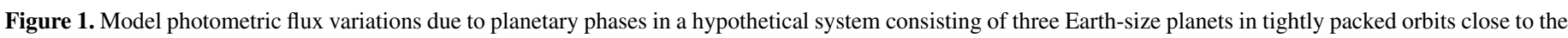
star and a Jupiter-size planet at 0.7 AU. The solid lines show the phase variations due to the individual planets and the dotted line indicates the combined effect.

\section{MULTI-PLANET SYSTEMS}

Here we describe the combination effects and decoupling issues for multi-planet systems.

\subsection{Combination Effects}

The discoveries from both radial velocity (RV) and transit exoplanet detections have revealed that planetary systems can come in a variety of orbital configurations and physical characteristics. Multiplicity appears to be a common trait of exosystems and thus we can expect most systems to exhibit phase signatures of more than one planet. The manner in which these signatures contribute to the total flux variations can cause an ambiguous interpretation of the data. Short-period planets can cause significant confusion if the observational cadence is insufficient to sample the variation cycle caused by the planets. For systems in which there is a dominant giant planet, the signatures of these smaller short-period planets can appear as correlated noise in the data depending on the photometric precision. In addition, it is unlikely that all the planets in a given system will transit their host star from the perspective of Earth, as we will see for the solar system in Section 4.2. Thus there may be additional unaccounted for signatures in the data if one is basing the model on those known to transit.

Shown in Figure 1 is an example model of flux variations due to the phase signatures of a four-planet system, the contribution from each planet shown as a solid line. The dominant source of the reflected light is due to a Jupiter-size planet in a circular orbit at $0.7 \mathrm{AU}$. There are also three Earth-size planets with periods less than 30 days. The combined signature is represented by a dotted line. Although one may detect the signal of the giant planet, the terrestrial planets will add correlated noise to the signal, the amplitude of which will depend on their respective albedos. The opposite effect is also true, that the phase signature of three known (from their transits) terrestrial planets will soon be distorted by the presence of an unknown (non-transiting) exterior giant planet.

\subsection{Fourier Decoupling}

For multi-planet systems, the planets are more likely to be in near-circular orbits than planets in single-planet systems (Wright et al. 2009). This circularization of the orbits is even more common for the tightly packed planetary orbits found for the Kepler systems (Moorhead et al. 2011). Thus, the combined phase signature of multi-planet systems may be adequately described as a sum of trigonometric functions suitable for Fourier analysis. Consideration of phase signatures for eccentric planets, such as those described by Kane \& Gelino (2010), may also be considered in this context using Keplerian techniques in the Fourier method. This is particularly important when evaluating the significance of peaks in the power spectrum and is discussed in detail by Cumming (2004), Cumming et al. (2008), and O'Toole et al. (2009).

The capability of a robust Fourier analysis to recognize the individual component planetary contributions to the total phase variations depends primarily on (1) the photometric precision, (2) the observational cadence, and (3) the duration of the observations. The observational cadence and duration limit the period analysis between the low fundamental frequency and the Nyquist frequency. A low signal-to-noise data set will produce peaks in the Fourier spectrum of similar power to spurious signals (aliases). An example is shown in Figure 2 where we have simulated a four-planet system with radii ranging from super-Earth $\left(2 R_{\oplus}\right)$ to large ice giants $\left(5 R_{\oplus}\right)$. The orbits are tightly packed with the outer planet in a $\sim 25$ day period orbit. The dotted line shows the combined phase variation for the entire system of planets. We have simulated data for 200 epochs spread over twice the period of the outer planet. Each time was passed through a Gaussian filter with a standard deviation of 15 minutes to randomize the times. A weighted Lomb-Scargle (L-S) period analysis (Lomb 1976; Scargle 1982) was applied using the methodology shown in Aharmin et al. (2005). This method applies inverse variance weighting calculated for each data point to each of the terms in the Fourier component. In cases where the error bars (and hence the weights) are equal, the weighted $\mathrm{L}-\mathrm{S}$ periodogram reduces to the standard $\mathrm{L}-\mathrm{S}$ periodogram. The bottom panel shows the periodogram for the simulated data with vertical dashed lines indicating the actual periods of the four-planet system.

There are a couple of issues to note regarding the Fourier disentanglement of the individual signatures depicted here. The planet located at $\sim 17$ days has the lowest phase amplitude, comparable to the noise properties of the data, and therefore is barely detectable. As such, improving either the cadence or the time baseline of the observations has little effect in improving the detection of this signature. The detection of the planet with the smallest period is only affected in so far as the cadence drops to a level that the orbital frequency becomes comparable to the Nyquist frequency. In this case, that situation would 

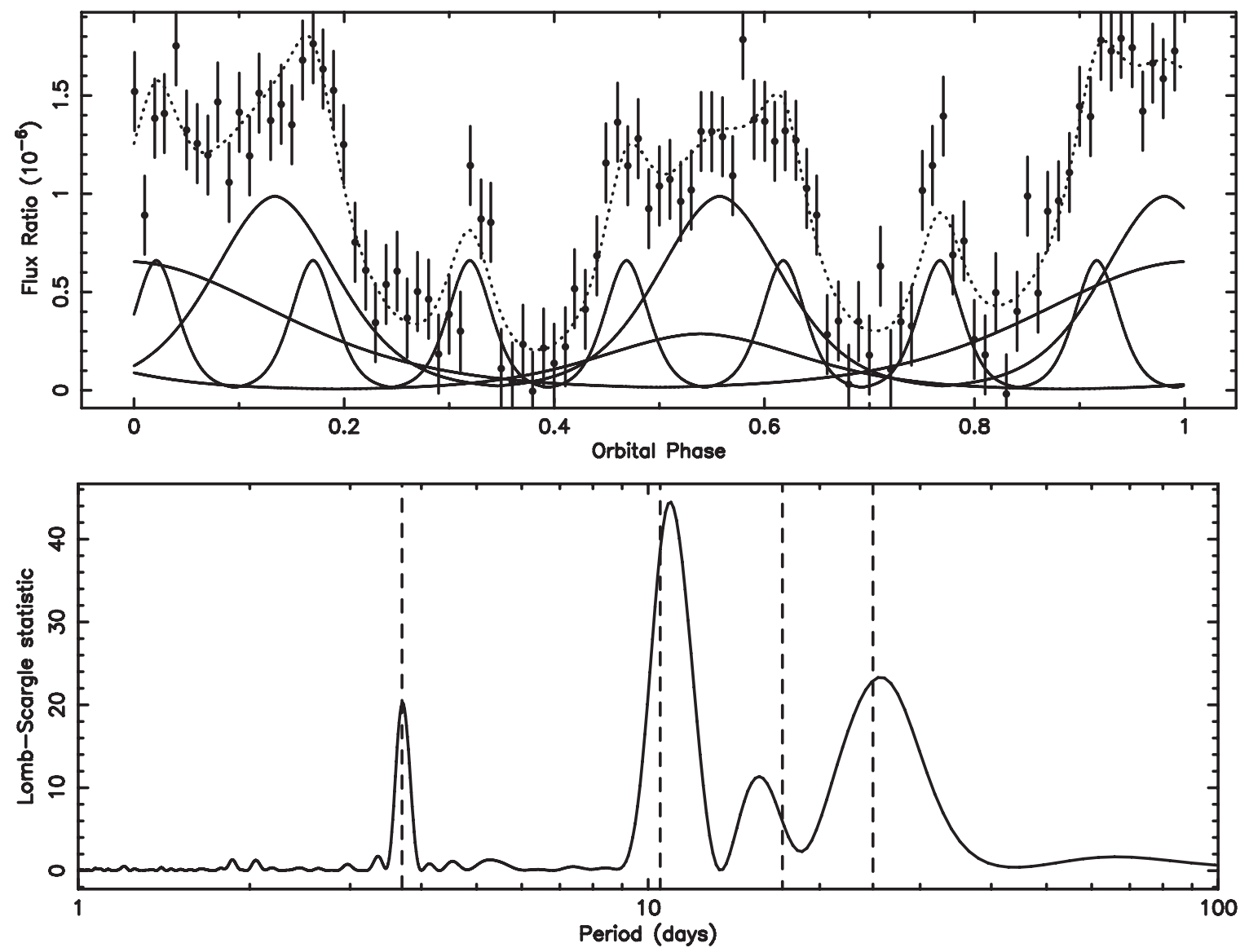

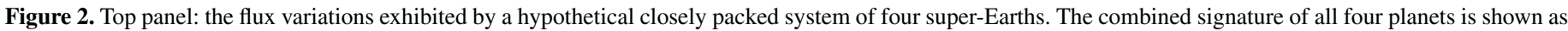

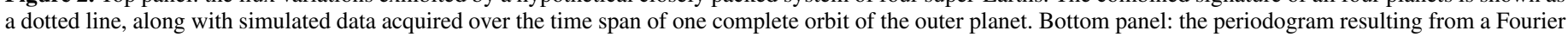
analysis of the data. The vertical dashed lines indicate the actual periods of the four planets.

result if the number of measurements dropped from 200 to 27 , yielding a cadence of $\sim 1.8$ days. One must consider this detection threshold when planning the observation strategy for a particular mission.

The Fourier method is thus suitable to extract such signatures in most cases except where the signal-to-noise is very low. Where one or more phase signatures dominate the signal, an iterative approach of subtracting those fitted signals and then re-analyzing the residuals can reveal the remaining planets. This method however does assume Gaussian noise with purely periodic signatures. Since the number of cycles is very limited, the maximum entropy method is an alternative approach since this is relatively efficient in detecting frequency lines with few assumptions regarding the initial estimates of the fit parameters (see, for example, Endl et al. 2000).

\section{THE SOLAR SYSTEM}

To find a classical case where phase variation confusion can occur, we need look no further than our own solar system. The data for the solar system planets were extracted from the JPL HORIZONS system. ${ }^{1}$

\subsection{Combined Phase Variations}

First we consider the total phase variations expected by the solar system when viewed along the ecliptic. Table 1 contains the

\footnotetext{
1 http://ssd.jpl.nasa.gov/?horizons
}

Table 1

Solar System Planetary Orbital Parameters and Peak Flux Ratios

\begin{tabular}{lrrcrrrrr}
\hline \hline Planet & \multicolumn{1}{c}{$\begin{array}{c}P \\
\text { (days) }\end{array}$} & $\begin{array}{c}c \\
(\mathrm{AU})\end{array}$ & $e$ & $\begin{array}{c}\omega \\
(\mathrm{deg})\end{array}$ & $\begin{array}{c}R_{p} \\
\left(R_{\oplus}\right)\end{array}$ & $\begin{array}{c}i \\
(\mathrm{deg})\end{array}$ & $A_{g}$ & $\begin{array}{c}\text { Flux Ratio } \\
\left(10^{-9}\right)\end{array}$ \\
\hline Mercury & 87.97 & 0.39 & 0.206 & 29.1 & 0.38 & 83.0 & 0.11 & 0.172 \\
Venus & 224.70 & 0.72 & 0.007 & 55.2 & 0.94 & 86.6 & 0.65 & 2.000 \\
Earth & 365.26 & 1.00 & 0.017 & 114.2 & 1.00 & 90.0 & 0.37 & 0.646 \\
Mars & 686.98 & 1.52 & 0.093 & 286.5 & 0.53 & 88.2 & 0.15 & 0.040 \\
Jupiter & 4332.82 & 5.20 & 0.048 & 275.1 & 11.22 & 88.7 & 0.52 & 4.836 \\
Saturn & 10755.70 & 9.54 & 0.054 & 336.0 & 9.46 & 87.5 & 0.47 & 0.880 \\
Uranus & 30687.15 & 19.19 & 0.047 & 96.5 & 4.01 & 89.2 & 0.51 & 0.037 \\
Neptune & 60190.03 & 30.07 & 0.009 & 265.6 & 3.89 & 88.2 & 0.41 & 0.013 \\
\hline
\end{tabular}

solar system orbital parameters used for these calculations. Note that when treating the solar system as an exosystem, one must be careful to distinguish between the longitude of perihelion and the argument of perihelion since the longitude of perihelion is the sum of the longitude of the ascending node and the argument of perihelion. Here we use the argument of perihelion $(\omega)$ derived from these quantities. From these parameters and the geometric albedos, we calculate the predicted phase variations whose amplitudes are also shown in Table 1.

Figure 3 shows these phase variations at two resolutions; one phased on the orbit of Neptune and the other phased on the orbit of Jupiter. The top panel is dominated by Jupiter, which has the largest phase amplitude. Uranus and Neptune have negligible contributions to the combined phase variations and Saturn produces a small modulation in the Jupiter signature. 

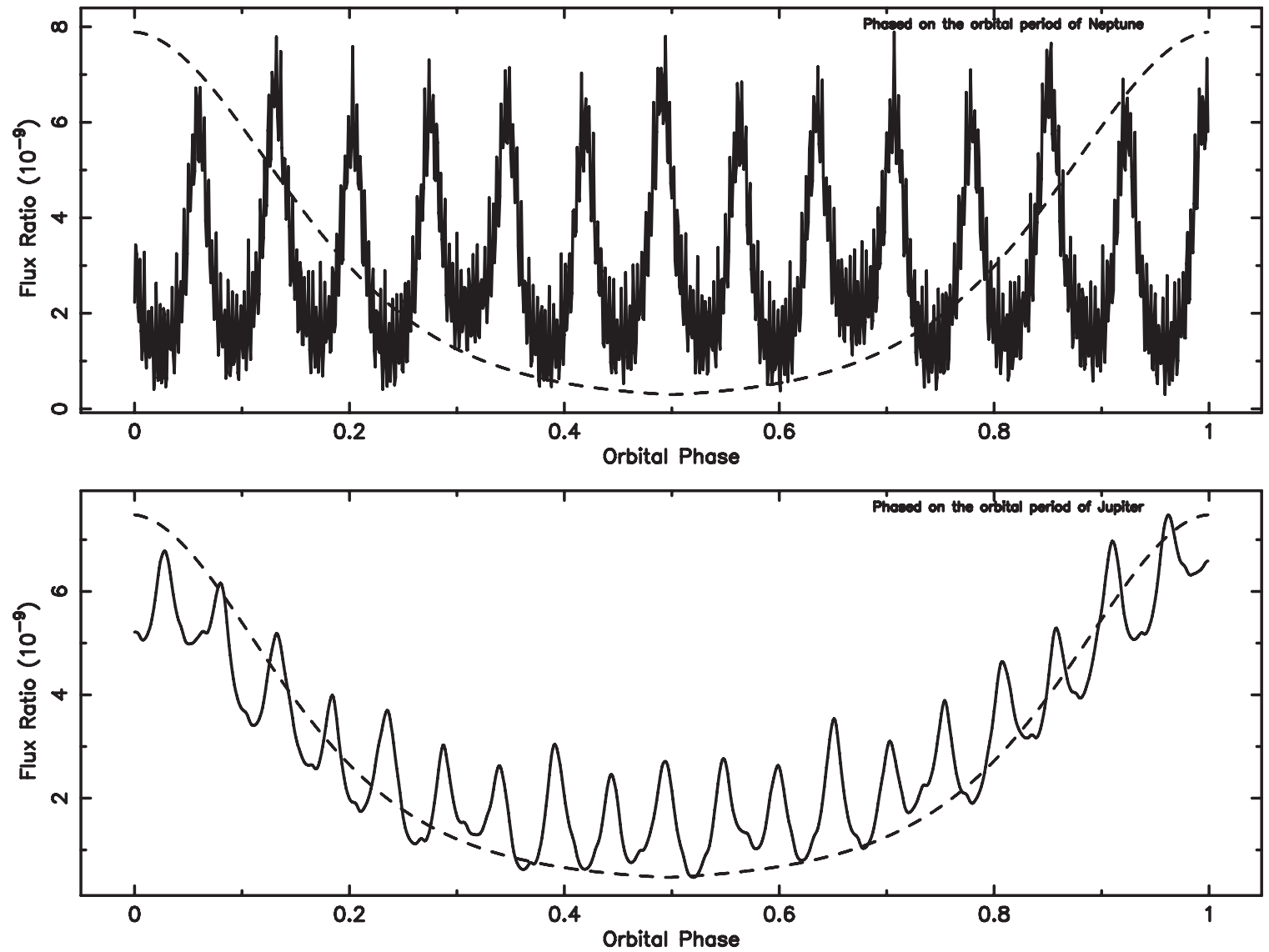

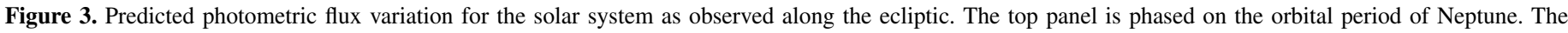

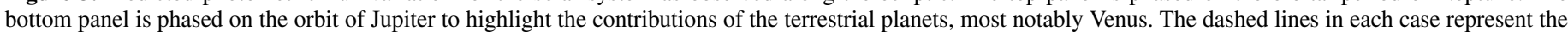
normalized phase function of Neptune and Jupiter, respectively.

However, the terrestrial planets are at least as significant in their contributions. Venus has a particularly large contribution, the amplitude of which is $43 \%$ that of Jupiter. The effects of the terrestrial planets can be seen much clearer in the lower panel, which shows the phase variations for one complete orbital period of Jupiter. Reaching the precision to detect the largest planet in our system is no guarantee that one would see the effects of the terrestrial planets as anything other than partially correlated noise. This is compounded by the relatively short orbital periods of the inner planets whose relation to the observation cadence may render those planets all but undetectable. The flux amplitudes for the solar system planets are extremely small, but this is an effect which can be scaled to alternative orbital configurations. We will see several examples of this in the following section discussing Kepler systems, where the predicted flux amplitudes are several orders of magnitude larger.

\subsection{Transit Probabilities}

It is worth noting that not all of the solar system would be detectable via the transit method, even if viewed along the ecliptic. The detection of the outer planets via either the transit or RV techniques is especially difficult, as described by Kane (2011). For a circular orbit, the geometric transit probability is inversely proportional to $a$, such that the inclination of the planet's orbital plane, $i$, must satisfy

$$
a \cos i \leqslant R_{p}+R_{\star} .
$$

By this criteria, observing our Sun along the ecliptic plane would only result in a transit of Earth. We performed a Monte Carlo simulation which rotates the plane of the solar system $\pm 10^{\circ}$ with respect to the observer. This simulation showed that there is only a very narrow range of viewing angles for which one would see more than one planet transit the Sun. Specifically, if the viewing angle departs from the ecliptic by between 1.76 and 1.78 , one will observe transits of both Mars and Neptune. This could be considered an extreme case of demonstrating that not all planets are necessarily accounted for in multi-planet transiting systems and thus the phase variations shown in Figure 3 will not be representative of what is predicted. However, the issue is still particularly pertinent for Kepler multi-planet systems which are in much more compact orbital configurations, as we will see in the following sections.

\section{KEPLER SYSTEMS}

Here we apply this methodology to several of the known multi-planet systems detected by the Kepler mission and discuss the impact on phase signatures.

\subsection{Kepler-20}

The Kepler-20 system consists of five known transiting planets which were discovered by Fressin et al. (2012) and Gautier et al. (2012). Three of the planets (b, c, and d) are of super-Earth size 1.91-3.07 $R_{\oplus}$ and the other two (e and f) are Earth size and smaller. The orbital and physical parameters for these planets are shown in Table 2. In the absence of information regarding the atmospheric properties of these planets, we have no knowledge of their geometric albedos. The potential diversity 

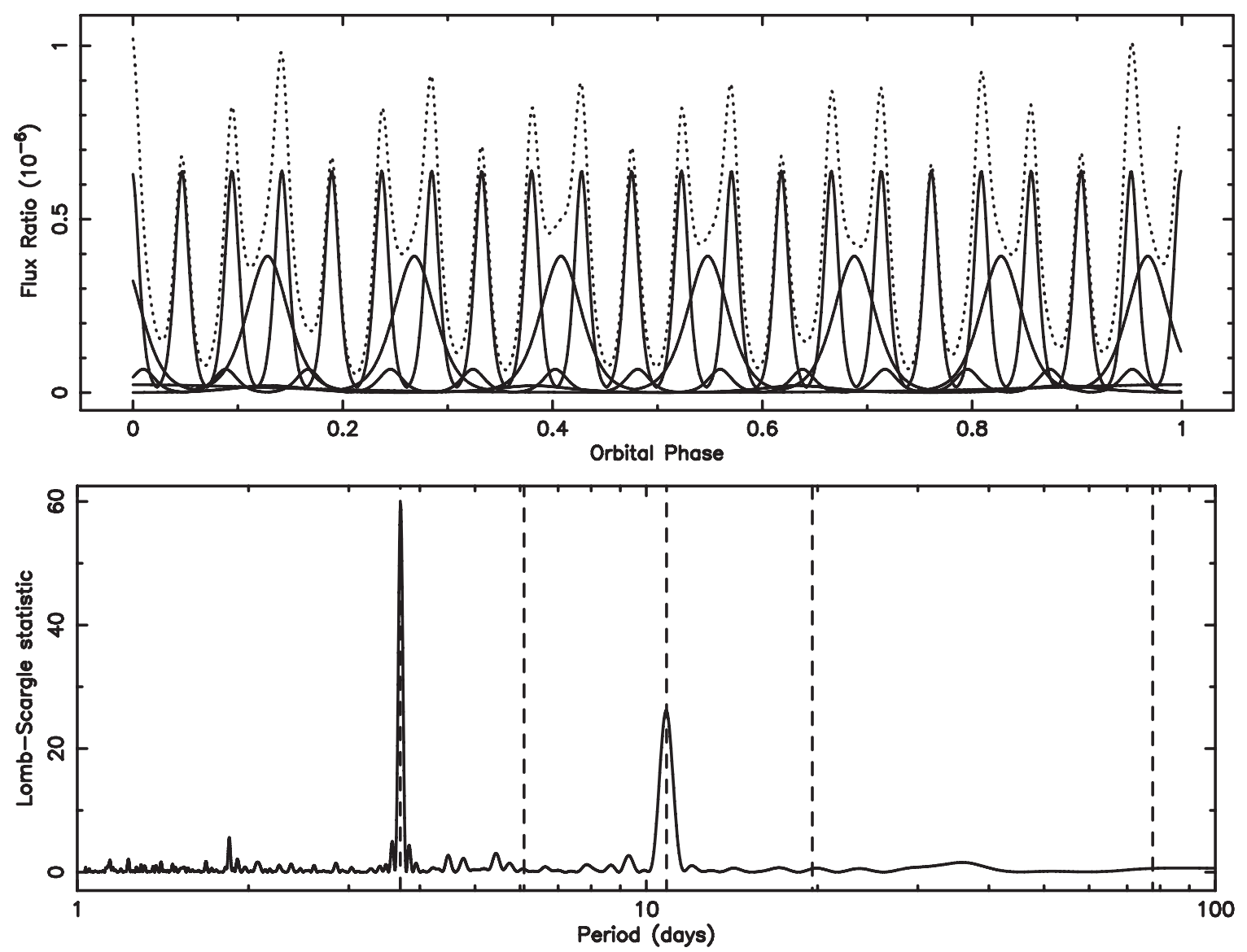

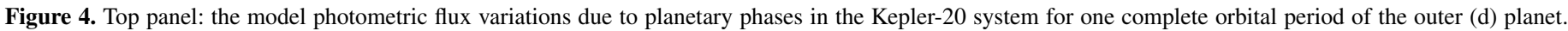

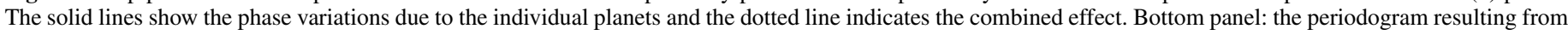
a Fourier analysis of the data. The vertical dashed lines indicate the actual periods of the five planets.

Table 2

Kepler-20 Planetary Orbital Parameters and Peak Flux Ratios

\begin{tabular}{lrccccc}
\hline \hline Planet & $\begin{array}{c}P \\
(\text { days })\end{array}$ & $\begin{array}{c}a \\
(\mathrm{AU})\end{array}$ & $\begin{array}{c}R_{p} \\
\left(R_{\oplus}\right)\end{array}$ & $\begin{array}{c}i \\
(\mathrm{deg})\end{array}$ & $A_{g}$ & $\begin{array}{c}\text { Flux Ratio } \\
\left(10^{-6}\right)\end{array}$ \\
\hline $\mathrm{b}$ & 3.696 & 0.045 & 1.91 & 86.50 & 0.2 & 0.639 \\
$\mathrm{c}$ & 10.854 & 0.093 & 3.07 & 88.39 & 0.2 & 0.394 \\
$\mathrm{~d}$ & 77.612 & 0.345 & 2.75 & 89.57 & 0.2 & 0.023 \\
$\mathrm{e}$ & 6.098 & 0.063 & 0.87 & 87.50 & 0.2 & 0.068 \\
$\mathrm{f}$ & 19.577 & 0.138 & 1.03 & 88.68 & 0.2 & 0.020 \\
\hline
\end{tabular}

of exoplanetary atmospheres, even among those of similar mass and semi-major axis, is sufficient to disqualify an assumption regarding the albedo of a planet. However, we are aware of an albedo dependence on star-planet separation (Sudarsky et al. 2005; Cahoy et al. 2010; Kane \& Gelino 2010), such as the case of HD 209458b which was determined to have an upper limit of $A_{g}<0.08$ from observations using the Microvariability and Oscillations of STars satellite (Rowe et al. 2008). For the purposes of this analysis, we assign a moderately low default value of $A_{g}=0.2$ to all planets so that we may evaluate the relative contributions of the other parameters to the overall flux. When performing the analysis for a given data set, these albedos may be treated as free parameters when identifying the shape of the phase signatures which match to the observed orbital periods from the transit photometry.

The resulting model phase variations for the Kepler-20 system are shown in the top panel of Figure 4, with the contribution from individual planets shown as solid lines and the total phase variations indicated by a dotted line. The two largest contributors to the phase variation are the $\mathrm{b}$ and $\mathrm{c}$ planets due to their larger size and proximity to the star, shown in Table 2 . The e planet has a smaller star-planet separation than the c planet but the phase amplitude is inhibited by its small size. However, were the e planet to have a substantial reflective atmosphere such as Venus, the amplitude of the phase variation for planet e would rise to become comparable to around half that of planet c. Thus the two smallest planets in this system may yet be distinguishable from the major sources of reflected light in the system.

The bottom panel of Figure 4 shows a sample periodogram using the techniques described in Section 3.2, where we have simulated data for the model phase curves shown in the top panel. The vertical dashed lines indicate the orbital periods of the known five planets in the system. As discussed earlier, in cases such as this where the phase signature is largely dominated by one or more planets, the remaining signatures may remain invisible regardless of the quality of the data or the time baseline of the observations. Thus we see that the signatures of the $b$ and $c$ planets, at 3.7 and 10.9 days, are unambiguously extracted from the data. One possible solution is to perform a fit to the these two phase signatures, subtracting them from the data, and then re-analyzing the residuals in an attempt to reveal presence of the remaining planets. The success of such an approach depends on the amplitude of the remaining phase signatures with respect to the noise properties of the data.

To investigate the potential for additional sources of phase amplitude components in the system, we re-analyze the Keck/HIRES RV data acquired for Kepler-20 by Gautier et al. 

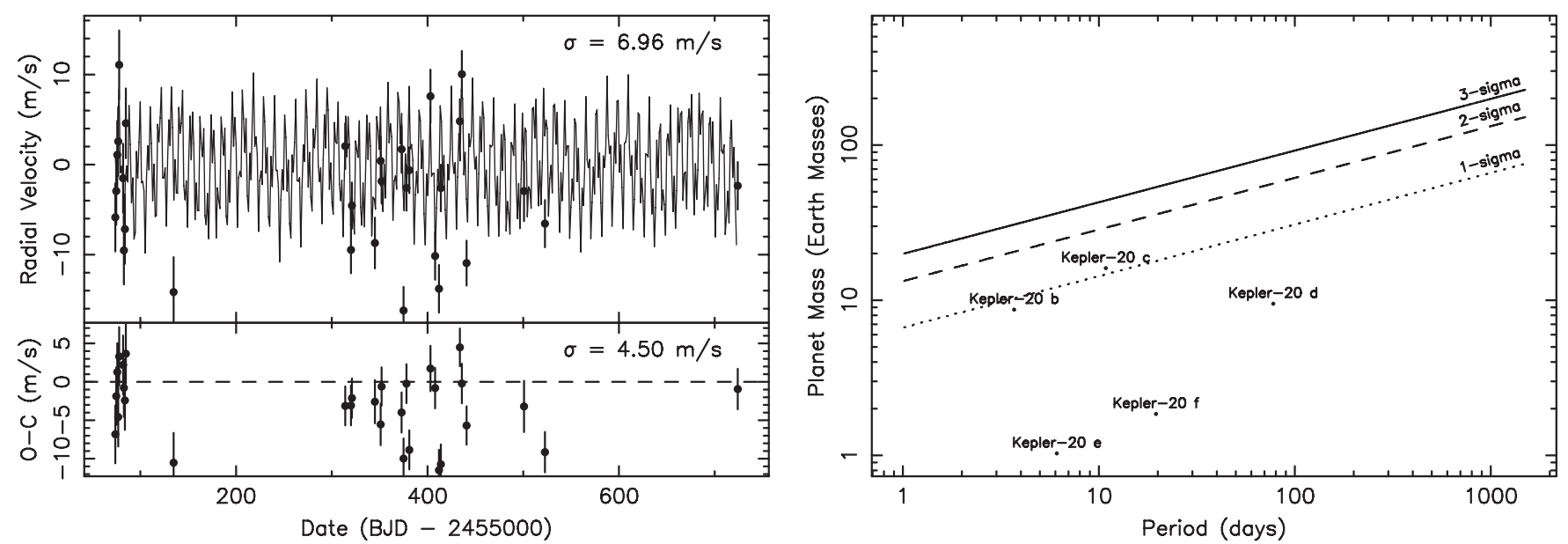

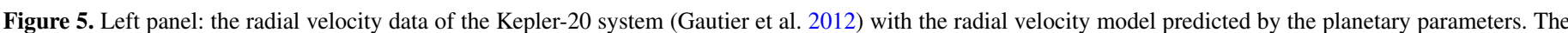

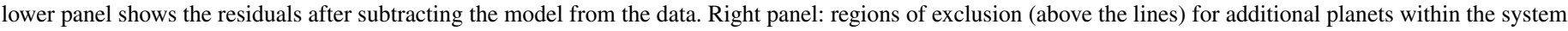
based upon the rms scatter of the residuals. The detected planets in the system are shown for reference.

(2012). In particular, we would like to investigate evidence of any additional companions in the system which may not necessarily transit the host star. Shown in the left panel of Figure 5 are the RV data along with the Keplerian model predicted by the planetary parameters described by Gautier et al. (2012) and the residuals to the fit shown at the bottom.

The mass of the d planet is difficult to constrain from the RV data and is described by Gautier et al. (2012) as having a $2 \sigma$ limit of $<20.1 M_{\oplus}$. The Markov Chain Monte Carlo analysis of the RV data yields a mass estimate for the d planet of $9.50_{-8.17}^{+6.23} M_{\oplus}$ (Geoff Marcy 2012, private communication). We adopt this value of the mass for deriving the Keplerian model for the data which then produces the shown residuals. The predicted $\mathrm{RV}$ semi-amplitudes for the e and $\mathrm{f}$ planets are $0.38 \mathrm{~m} \mathrm{~s}^{-1}$ and $0.47 \mathrm{~m} \mathrm{~s}^{-1}$, respectively, and therefore have a negligible contribution to the model.

The residuals shown in Figure 5 still contain a slightly reduced rms scatter of $4.5 \mathrm{~m} \mathrm{~s}^{-1}$. We calculate RV amplitudes that equal this $1 \sigma$ scatter as a function of orbital period and planet mass. These are shown in the right panel of Figure 5, with the lines representing multiples of the rms scatter of the residuals. One consequence of this figure is that we can exclude the presence of a Jupiter-mass planet in the system out to a period of $\sim 1000$ days at the $3 \sigma$ level. The exclusion yields significant information for a correct model of the phase variations since a Jupiter-size planet with a 200 day period would produce a long-term trend in the photometry, where the amplitude is similar to that of the c planet.

\subsection{Kepler-33}

Kepler-33 is a system of five known transiting planets which was announced by Lissauer et al. (2012). The complete parameters for these planets are shown in Table 3, where we have once again adopted fiducial albedo values of 0.2 . This system is quite different from the Kepler-20 system since it consists of planets in a size regime ranging from super-Earth to Neptune and a compact period range between 5 and 42 days. The major consequence of this compact planetary configuration is that all of the detected planets contribute comparable amounts of flux to the total phase variations of the system. For example, since the outer planets have a larger size, it somewhat compensates
Table 3

Kepler-33 Planetary Orbital Parameters and Peak Flux Ratios

\begin{tabular}{lrccccc}
\hline \hline Planet & $\begin{array}{c}P \\
\text { (days) }\end{array}$ & $\begin{array}{c}a \\
(\mathrm{AU})\end{array}$ & $\begin{array}{c}R_{p} \\
\left(R_{\oplus}\right)\end{array}$ & $\begin{array}{c}i \\
(\mathrm{deg})\end{array}$ & $A_{g}$ & $\begin{array}{c}\text { Flux Ratio } \\
\left(10^{-6}\right)\end{array}$ \\
\hline $\mathrm{b}$ & 5.668 & 0.068 & 1.74 & 86.39 & 0.2 & 0.238 \\
$\mathrm{c}$ & 13.176 & 0.119 & 3.20 & 88.19 & 0.2 & 0.262 \\
$\mathrm{~d}$ & 21.776 & 0.166 & 5.35 & 88.71 & 0.2 & 0.375 \\
$\mathrm{e}$ & 31.784 & 0.214 & 4.02 & 88.94 & 0.2 & 0.128 \\
$\mathrm{f}$ & 41.029 & 0.254 & 4.46 & 89.17 & 0.2 & 0.112 \\
\hline
\end{tabular}

for their larger star-planet separation. The phase variations are shown in the top panel of Figure 6, where the solid lines indicate the individual planets and the dotted line represents the combined effect. The similar phase amplitudes and small range of periods result in a significantly improved Fourier decoupling of the phase signatures compared with the Kepler-20 system. The bottom panel of Figure 6 shows the periodogram from a simulated data set constructed from the phase curves shown in the top panel. The inner three planets are recovered with ease due to their relatively large phase amplitudes. The outer two planets have detectable signatures from the combined analysis of all five planets but would benefit from the prior extraction of the three dominant planets, as discussed earlier.

RV data are not available for Kepler-33 at the time of writing, which prevents the exclusion of additional planets as was performed for the Kepler-20 system. For such a compact system, it is not unreasonable to postulate the existence of further nontransiting planets in the system. This could have a profound effect on the combined phase variations if Jovian-size planets lie just beyond the reach of the transit method to detect them. An example of such a case is shown in Figure 7. We have added the signature (shown as a dashed line) of a non-transiting Jupiter-size planet with an orbital period of 55 days. This planet immediately dominates the phase variations with an amplitude which is almost a full order of magnitude larger than the previous largest amplitude of the c planet. The signatures of the inner planets are thus reduced to correlated noise in the face of this new dominant signature, making extraction more difficult since the albedos of all of the planets are treated as free parameters in the fit to the data. 

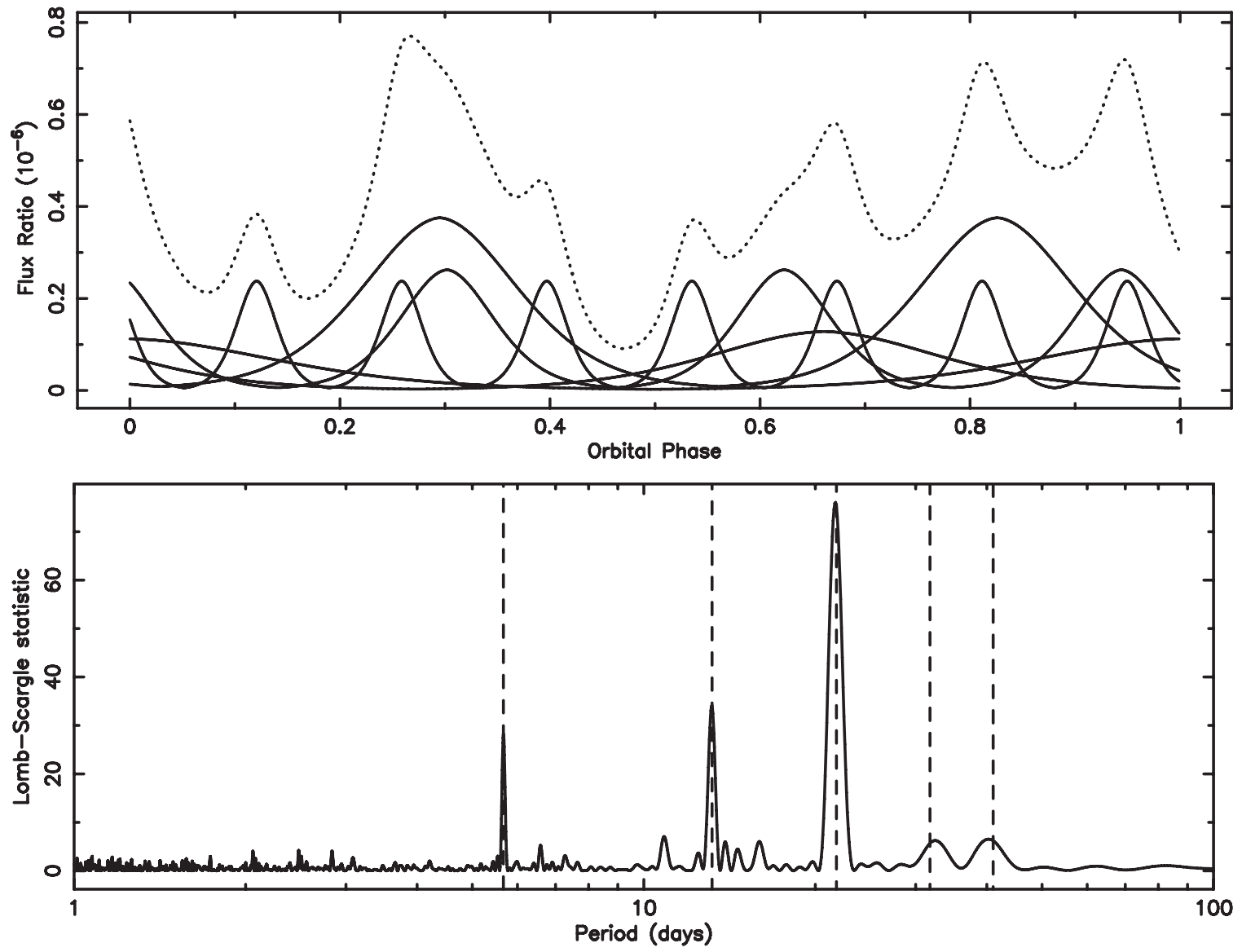

Figure 6. Top panel: the model photometric flux variations due to planetary phases in the Kepler-33 system for one complete orbital period of the outer (f) planet. The solid lines show the phase variations due to the individual planets and the dotted line indicates the combined effect. Bottom panel: the periodogram resulting from a Fourier analysis of the data. The vertical dashed lines indicate the actual periods of the five planets.

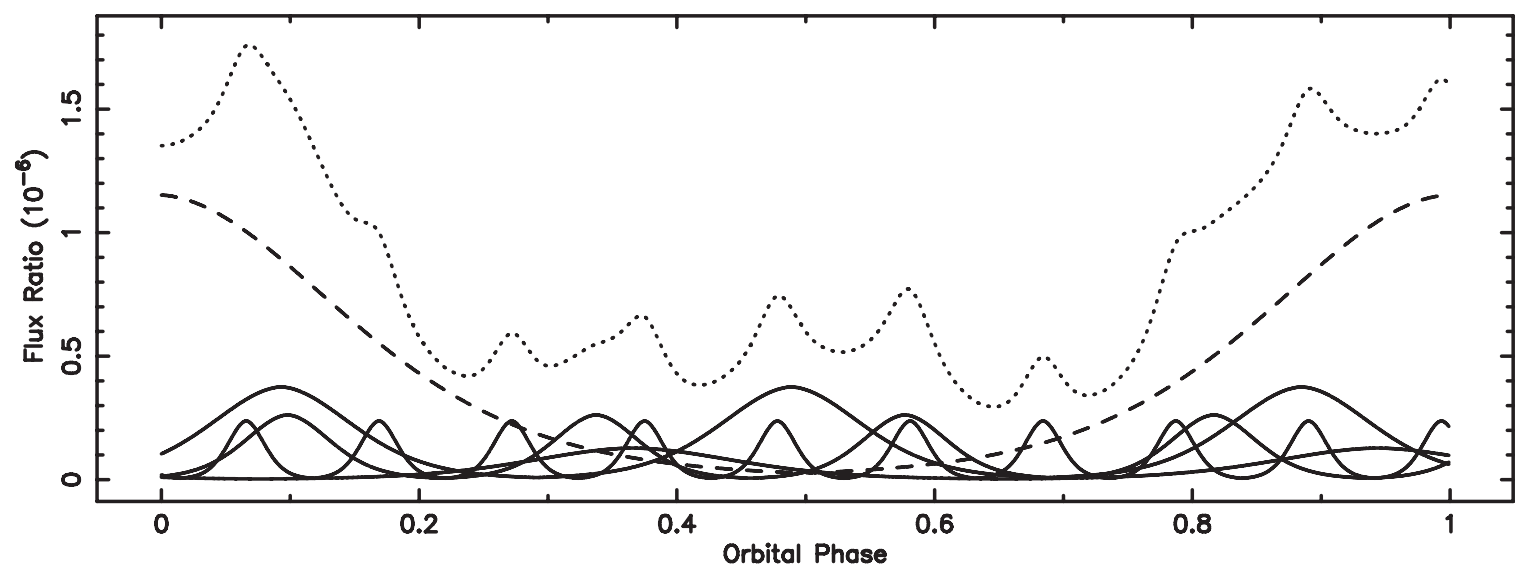

Figure 7. As for the top panel of Figure 6 except that an additional non-transiting planet has been added with a period of 55 days, indicated by the dashed line. The plot now shows one complete orbital period of this new planet.

\section{CONCLUSIONS}

Initial results from the Kepler mission indicate that multiplanet systems are relatively common. The precision of the Kepler mission and future photometric studies will allow detailed investigations of photometric variations due to planetary phase signatures. We have shown that the various phase signatures in these systems can be combined to produce an emulation of correlated noise in the photometry. This confusion can both cause the planets to be indistinguishable from each other and disguise the phase signature from the dominant planet in the system. On the other hand, non-transiting planets, usually at star-planet separations beyond the known size of the system, may either introduce new sources of correlated noise or indeed be the dominant source of variations in the system, such as was demonstrated for Kepler-33.

We have shown that Fourier analysis of the data will be able to extract many signatures from multi-planet systems, but depends critically upon the cadence and duration of the observations in order for the frequency distribution of the signatures to be 
properly sampled. In practice, the largest hindrance will be that of the photometric precision, which will prevent even highfrequency signals from being detected. Our investigation of the solar system demonstrates an example of confusion sources, where the signals of Jupiter and Venus may be extracted but the signatures of the remaining planets are unlikely to be interpreted as anything other than correlated noise. It is worth noting that the solar system would not be detected as a multi-planet system due to the misalignment of the planetary orbital planes, with the exception of Mars and Neptune. We further demonstrate the difficulties in deciphering the phase variation for the Kepler-20 and Kepler-33 systems. The interesting aspect of these systems, along with many other Kepler multi-planet systems, is the compact nature of the orbits such that it is possible to observe complete orbital phases for all planets within a reasonable time frame. One corollary of this is that there is great potential for a non-transiting giant planet to exist beyond the known planets in each system whose signature would significantly change the predicted phase variations of the system.

We have focused solely on the photometric variations due to the phase functions of the planets. There are, however, numerous other effects, mentioned in Section 1, which need to be accounted for before one can begin to examine the signals discussed here. With further observations of the host stars and an extended baseline of precision photometry, it is hoped that we can eventually disentangle the various contributions to the observed flux from these most interesting systems.

The authors would like to thank Geoff Marcy, Nick Gautier, Natalie Hinkel, and Diana Dragomir for their useful suggestions and comments. We would also like to thank the anonymous referee, whose comments greatly improved the quality of the paper.

\section{REFERENCES}

Aharmin, B., Ahmed, S. N., Anthony, A. E., et al. 2005, PhRvD, 72, 052010 Batalha, N. M., et al. 2012, ApJS, submitted (arXiv:1202.5852)

Borucki, W. J., Koch, D. G., Basri, G., et al. 2011a, ApJ, 728, 117

Borucki, W. J., Koch, D. G., Basri, G., et al. 2011b, ApJ, 736, 19

Cahoy, K. L., Marley, M. S., \& Fortney, J. J. 2010, ApJ, 724, 189

Cumming, A. 2004, MNRAS, 354, 1165

Cumming, A., Butler, R. P., Marcy, G. W., et al. 2008, PASP, 120, 531

Endl, M., Kürster, M., \& Els, S. 2000, A\&A, 362, 585

Fressin, F., Torres, G., Rowe, J. F., et al. 2012, Natur, 482, 195

Gautier, T. N., III, Charbonneau, D., Rowe, J. F., et al. 2012, ApJ, 749, 15

Jackson, B. K., Lewis, N. K., Barnes, J. W., et al. 2012, ApJ, 751, 112

Kane, S. R. 2011, Icar, 214, 327

Kane, S. R., \& Gelino, D. M. 2010, ApJ, 724, 818

Kane, S. R., \& Gelino, D. M. 2011, ApJ, 729, 74

Kane, S. R., \& Gelino, D. M. 2012, MNRAS, 424, 779

Lissauer, J. J., Fabrycky, D. C., Ford, E. B., et al. 2011a, Natur, 470, 53

Lissauer, J. J., Marcy, G. W., Rowe, J. F., et al. 2012, ApJ, 750, 112

Lissauer, J. J., Ragozzine, D., Fabrycky, D. C., et al. 2011b, ApJS, 197, 8

Lomb, N. R. 1976, Ap\&SS, 39, 447

Mazeh, T., Nachmani, G., Sokol, G., Faigler, S., \& Zucker, S. 2012, A\&A, 541,56

Moorhead, A. V., Ford, E. B., Morehead, R. C., et al. 2011, ApJS, 197, 1

O’Toole, S. J., Tinney, C. G., Jones, H. R. A., et al. 2009, MNRAS, 392, 641

Pfahl, E., Arras, P., \& Paxton, B. 2008, ApJ, 679, 783

Rowe, J. F., Matthews, J. M., Seager, S., et al. 2008, ApJ, 689, 1345

Scargle, J. D. 1982, ApJ, 263, 835

Shporer, A., Jenkins, J. M., Rowe, J. F., et al. 2011, AJ, 142, 195

Sudarsky, D., Burrows, A., Hubeny, I., \& Li, A. 2005, ApJ, 627, 520

Welsh, W. F., Orosz, J. A., Seager, S., et al. 2010, ApJL, 713, 145

Wright, J. T., Upadhyay, S., Marcy, G. W., et al. 2009, ApJ, 693, 1084 